\title{
Relações entre metodologia ativa, avaliação formativa e aprendizagem discente no curso de engenharia mecânica
}

\section{Relationships among active methodology, formative assessment and student learning in the mechanical engineering course}

\author{
1 Rodolfo Enrique Perdomo Freitas rodolfo.perdomo@utp.br \\ 2 Maria Iolanda Fontana \\ ${ }^{3}$ Angela Helena Zatti
}

1 Engenheiria Mecânica (Universidad de la Republica, Uruguai) e Especialista em Gestão de Empresa de Manutenção de
Aeronaves (UTP), Professor do curso de Engenharia Mecânica da Universidade Tuiuti do Paraná - UTP.
2 Pedagoga, Mestre e Doutora em Educação. Professora do Programa de Pós-graduação em Educação da Universidade Tuiuti
do Paraná - UTP.
3 Mestre em Comunicação e Linguagens (UTP), Professora do curso de Engenharia Mecânica da Universidade Tuiuti do Paraná.

\section{RESUMO}

O presente trabalho trata de uma pesquisa-ação que investiga a relação entre metodologias ativas e avaliação formativa da aprendizagem no ensino da disciplina de Fundamentos da Engenharia Mecânica Automobilística FEMA - do curso de graduação de Engenharia Mecânica da Universidade Tuiuti do Paraná. As metodologias ativas utilizadas foram Peer Instruction (PI) - Instrução por Pares, Writing Across the Curriculum (WAC) - Escrita por todo o Currículo e - Problem Based Learning (PBL) - Aprendizagem Baseada em Problemas. Apresenta-se a proposta metodológica adotada no desenvolvimento da disciplina e, também, como foi avaliada a aprendizagem dos alunos em função dos objetivos de ensino. Essa proposta tem como fundamento a perspectiva formativa da avaliação da aprendizagem, de característica investigativa e democratizadora, presente no debate sobre o tema. Descreve-se a realização das avaliações e acompanhamento diagnóstico e interventivo, por meio de uma planilha para a visualização pelo professor do desempenho discente e, quando necessário, a revisão do ensino e da metodologia. Finalmente, se faz uma breve análise sobre os resultados, destacando que vincular metodologias ativas com a avaliação diagnóstica, contínua e formativa elevou a aprendizagem e reduziu a repetência e a evasão discente, como também alertou para a necessidade de interdisciplinaridade e contextualização dos conteúdos na disciplina.

\section{Palavras-chave}

Avaliação formativa. Educação superior. Metodologia ativa. Pesquisa-ação. Prática pedagógica.

\begin{abstract}
The present work is an action research that investigates the relationship between active methodologies and formative assessment of learning in the teaching of Fundamentals of Automobile Mechanical Engineering - FEMA - in the Mechanical Engineering undergraduate course at University Tuiuti do Paraná. The active methodologies chosen for application were Peer Instruction, WAC Writing Across the Curriculum - and PBL - Problem Based Learning. The methodological proposal adopted in the development of the subject is presented, as well as how the students' learning was evaluated according to the teaching objectives. This proposal is based on the formative perspective of learning assessment with an investigative and democratizing characteristic, presented in the debate on the theme. The performance of the evaluations and diagnostic and interventional follow-up is described using a spreadsheet for the professor to visualize the student's performance and, when necessary, to review the teaching and the methodology. Finally, a brief analysis on the results is made, highlighting that linking active methodologies with diagnostic, continuous and formative assessment increased learning and reduced student repetition and dropout as well as alerting to the need of interdisciplinarity and contextualization of the contents in the subject.
\end{abstract}

\section{Keywords}

Formative evaluation. College education. Active methodology. Action research. Pedagogical practice.

\section{Como você deve citar?}

FREITAS, Rodolfo Enrique Perdomo; FONTANA, Maria lolanda; ZATTI, Angela Helena. Relações entre metodologia ativa, avaliação formativa e aprendizagem discente no curso de engenharia mecânica. Cadernos UniFOA, Volta Redonda, n. 45, p.97-106, abril 2021. 


\section{INTRODUÇÃO}

A universidade Tuiuti do Paraná, membro do Consórcio STHEM/Brasil ${ }^{4}$ (do inglês, Science, Technology, Humanity, Engineering and Mathematics), desde o ano de 2015, organizou a formação de seus professores para o estudo e pesquisa sobre metodologias ativas em sala de aula. Essa iniciativa está integrada ao processo de formação continuada dos professores da Universidade, desenvolvida pelo Núcleo de Apoio ao Docente (NAD) que, entre outras ações, desenvolve com os professores de diferentes cursos da graduação, interessados em investigar a própria prática pedagógica, o projeto de pesquisa "Relações entre as Inovações Pedagógicas e a Aprendizagem Discente nos Cursos de Graduação da Universidade Tuiuti do Paraná". O projeto tem o objetivo de avaliar a aprendizagem dos estudantes da graduação da Universidade mediada pelo uso de metodologias ativas em sala de aula e, ao mesmo tempo, promover a inovação das práticas pedagógicas de docentes dos cursos de graduação da Universidade Tuiuti do Paraná.

O presente trabalho resulta dos estudos realizados no referido grupo de pesquisa na implantação de uma proposta de avaliação formativa, contínua e diagnóstica aplicada em alguns períodos do curso de graduação em Engenharia Mecânica. A metodologia utilizada é a pesquisa-ação, desenvolvida conforme os passos metodológicos criados por Thiollent (1998), que tem como objetivo aclarar e diagnosticar uma situação; formular estratégias de ação; desenvolver essas estratégias e avaliar sua eficiência; ampliar a compreensão da nova situação (situação resultante). O problema sob observação é a reprovação, evasão e dificuldades de aprendizagem em algumas disciplinas do curso de Engenharia Mecânica. As variáveis analisadas no processo da pesquisa-ação referem-se às repercussões da articulação entre as metodologias ativas e avaliação formativa para o desenvolvimento e a aprendizagem dos discentes.

Apresenta-se o encaminhamento da pesquisa realizada na disciplina de Fundamentos da Engenharia Mecânica Automobilística (FEMA), ministrada no primeiro período do curso, durante o semestre letivo 2019, em 80 horas/aula, com 34 alunos matriculados na disciplina. A proposta utilizou a combinação de diferentes metodologias ativas articuladas à perspectiva formativa, diagnóstica e contínua de avaliação com o objetivo de motivar os alunos para as aulas e melhorar a aprendizagem geral da turma. Os discentes foram avaliados em suas competências e habilidades, segundo o previsto no plano de ensino. 0 processo avaliativo foi planejado e executado em todas as aulas e acompanhado por meio de uma planilha com os registros do desempenho dos discentes para, com base nesses dados, o professor (re)planejar as intervenções articuladas referentes à metodologia da aula e à avaliação da aprendizagem.

A avaliação na perspectiva formativa busca a superação da prática classificatória tradicional que exclui o estudante do processo constante de aprendizagem e de seu desenvolvimento, pois tem como premissa que todos, mesmo com experiências escolares e ritmos diferentes, são capazes de aprender, desde que assegurada a mediação didática pertinente às suas necessidades. Nesse contexto, a avaliação formativa assume a dimensão democrática do trabalho educativo. A proposta avaliativa, articulada às metodologias ativas que são apresentadas neste artigo, tem essa perspectiva, ou seja, está efetivamente interessada nas aprendizagens dos discentes, como também em encontrar melhores métodos para o que está sendo ensinado e não apenas a verificação técnica da aprendizagem para aprovação ou reprovação.

Antes de apresentar o desenvolvimento da proposta avaliativa desenvolvida na referida disciplina do curso de Engenharia Mecânica, são feitas algumas considerações sobre o entendimento de avaliação formativa e das metodologias ativas utilizadas que embasam os estudos e análises desta pesquisa.

4 O objetivo do consórcio STHEM/Brasil é promover inovações pedagógicas para a melhoria do ensino e da aprendizagem nas áreas de Ciência, Tecnologia, Humanidades, Engenharia e Matemática. 


\section{REFERENCIAL TEÓRICO}

A avaliação da aprendizagem na educação superior tem sido objeto da pesquisa educacional, principalmente nas últimas décadas, considerando a contribuição dessa dimensão da prática pedagógica para promover a democratização da formação profissional nesse nível de ensino. 0 perfil discente tem desafiado professores a repensarem sua ação educativa que envolve, além do ensino de conteúdos da área que ensina, a formação do profissional crítico, ético, criativo e responsável com a transformação de relações excludentes que ocorrem na sociedade capitalista.

O mapeamento da produção do conhecimento sobre as avaliações das aprendizagens no Brasil, realizado por Boldarine, Barbosa e Annibal (2017), no período 2010 a 2014, em vários periódicos de circulação nacional, revela ser comum a preocupação com práticas avaliativas que contribuem nos processos de aprendizagem dos alunos e aponta que há pouca discussão sobre como preparar os docentes para as práticas avaliativas, tanto na graduação como na formação continuada. Os autores também observam a ausência de um debate sobre a organização dos acordos pedagógicos que ocorrem nas salas de aula na realização dos processos avaliativos.

Portanto, investigar sobre qual a melhor forma de avaliar os estudantes da educação superior é complexo e, ao mesmo tempo, necessário, pois não se resume apenas em discutir métodos que podem ser utilizados, segundo apenas suas características, mas também é preciso discutir que tipo de aprendizagem eles promovem e para quais objetivos formativos.

O presente trabalho pretende contribuir com o fomento ao debate, ao apresentar os dados da proposta avaliativa desenvolvida a partir do processo de formação continuada na universidade, realizada por meio da pesquisa-ação, e também ao analisar a perspectiva formativa da avaliação, sistematizada em atividades avaliadas continuamente como subsídio diagnóstico para intervenção nas dificuldades verificadas.

No Brasil, autores que discutem a avaliação utilizam várias expressões para conceber a perspectiva formativa dessa prática, tais como: mediadora, emancipatória, dialógica. Independentemente da terminologia usada, os posicionamentos desses autores, tais como Luckesi (2008; 2018), Hoffman (2009) e Vasconcellos (2010), pertencem a uma mesma visão de avaliação que contrapõe a perspectiva positivista, classificatória e seletiva do ato de avaliar a aprendizagem, portanto excludente.

Para os autores que discutem a perspectiva formativa da avaliação, como Villas Boas (2009), o elemento-chave da avaliação formativa é o feedback, ou seja, a informação sobre a distância entre o nível de desenvolvimento alcançado pelo estudante e aquele fixado como objetivo do trabalho educativo. A avaliação formativa inclui informações qualitativas sobre o feedback (entre docente e discente) e automonitoramento (percepção da aprendizagem pelo discente). Isso significa que o desenvolvimento da capacidade de automonitoramento compõe o conjunto de aprendizagens a serem adquiridas (SILVA; MENDES, 2017, p. 283).

Conforme explica Garcia (2009, p. 211), no processo avaliativo, "as escolhas exercidas pelos professores podem ampliar ou limitar as oportunidades para que os estudantes demonstrem o que aprenderam" e estas podem ou não fornecer feedback ao aluno sobre seu desempenho durante o processo de ensino-aprendizagem.

Defende-se uma proposta que utilize diferentes métodos de avaliação, de acordo com os objetivos de ensino e o desenvolvimento de competências e habilidades. Assim, valoriza-se uma concepção de avaliação contínua e diagnóstica de caráter investigativo e formativo que ofereça o feedback sobre a aprendizagem do discente para subsidiar o (re)planejamento do processo de ensino. Entende-se que as avaliações praticadas pelo professor de modo intencional e consciente permitem tomar decisões fundamentais para o aprendizado individual e coletivo. 
Segundo Luckesi (2018, p. 91), o papel diagnóstico da avaliação é "revelar a qualidade da aprendizagem dos estudantes nos sucessivos momentos de sua atividade de aprender, subsidiando decisões constantes por parte do gestor da sala de aula (professor), tendo em vista atingir o resultado esperado". Significa um olhar construtivo sobre a aprendizagem e que, para atingir o nível de aprendizagem satisfatório, o discente necessitará da ajuda e da mediação do professor. Para Luckesi (2018, p.112-113), o uso diagnóstico dos resultados da avaliação subsidia a prática pedagógica consistente e transforma a avaliação em parceira de professores e estudantes que informam o que aprenderam ou necessitam aprender.

O uso diagnóstico dos resultados da avaliação, ênfase da proposta avaliativa assumida na disciplina de FEMA, tem o posicionamento em favor da inclusão de todos os discentes, no processo de construção e apropriação de conhecimentos fundamentais para a formação do profissional competente e de responsabilidade social. Portanto, a avaliação do ponto de vista epistemológico, conforme argumenta Luckesi (2018, p.28), "implica 'uma posição de não-indiferença' frente ao seu objeto de investigação, o que quer dizer que, nesse campo de conhecimento, não existe neutralidade". Essa é a perspectiva que orientou a proposta avaliativa assumida no projeto de pesquisa-ação, associada às metodologias ativas Peer Instruction (PI) - Instrução por Pares, Writing Across the Curriculum (WAC) - Escrita por todo o Currículo e - Problem Based Learning (PBL) - Aprendizagem Baseada em Problemas.

A ênfase na metodologia Peer Instruction (PI) (ou Instrução por Pares), na disciplina de FEMA, deve-se principalmente pela interação entre discentes e pelo feedback avaliativo do professor para dirimir dificuldades e elevar a aprendizagem de todos. 0 método criado para o ensino de Física, em 1991, pelo professor Eric Mazur ${ }^{5}$, foi adaptado na disciplina de FEMA, seguindo as etapas: leitura prévia pelos alunos sobre o conteúdo e, no decorrer da aula, resposta às questões; no momento das respostas, o professor já identifica se os alunos compreenderam ou não o conteúdo; há a promoção de um debate para as questões identificadas como menos respondidas - ou menos compreendidas - e aqueles estudantes que detêm tal conhecimento têm a oportunidade de explicar aos colegas com dúvidas. Ao contrário da prática comum de fazer perguntas informais, durante uma aula tradicional, que normalmente envolve uns poucos alunos altamente motivados, a metodologia do Peer Instruction pressupõe questionamentos mais estruturados e que envolvem todos os alunos na aula. Nas aulas de FEMA, a discussão do conteúdo busca promover a transmissão/assimilação dos conhecimentos entre os alunos presentes, ou seja, entre os pares.

A metanálise da produção de conhecimento acerca dos impactos da adoção do Peer Instruction, apresentada por Müeller et al. (2017), revela uma diversidade de resultados que mostra que a metodologia, "é uma proposta altamente recomendada, especialmente quando se busca engajar o estudante no processo de aprendizagem, bem como melhorar seus resultados de aprendizagem"(MÜELLER et al., 2017, p. 17-18).

Outra metodologia utilizada nas aulas de FEMA foi o WAC - Writing Across the Curriculum ${ }^{6}$ (Escrever através das disciplinas ou escrita por todo o Currículo). Trata-se de uma metodologia que tem o objetivo

5 Síntese das etapas da proposta de Mazur para o Pl: 1. Uma curta apresentação oral sobre os elementos centrais de um dado conceito ou teoria feita por cerca de 20 minutos. 2. Uma pergunta de múltipla escolha, geralmente conceitual, denominada Teste Conceitual, é colocada aos alunos sobre o conceito (teoria) apresentado na exposição oral. 3. Os alunos têm entre um e dois minutos para pensarem silenciosamente sobre a questão apresentada. 4. Os estudantes registram suas respostas individualmente e as mostram ao professor, usando algum sistema de respostas (por ex., clickers ou flashcards). 5. De acordo com a distribuição de respostas, o professor pode passar para o passo seis (quando a frequência de acertos está entre 35\% e 70\%), ou diretamente para o passo nove (quando a frequência de acertos é superior a 70\%). 6. Os alunos discutem a questão com seus colegas por um a dois minutos. 7. Os alunos registram suas respostas revisadas e as mostram ao professor, usando o mesmo sistema de respostas do passo 4. 8. O professor tem um retorno sobre as respostas dos alunos a partir das discussões e pode apresentar os resultados para os alunos. 9. O professor, então, explica a resposta da questão aos alunos e pode apresentar uma nova questão sobre o mesmo conceito ou passar ao próximo tópico da aula, voltando ao primeiro passo (MAZUR,1997, p.22-24 apud MÜELLER 2017, p.2-3).

6 Writing Across the Curriculum (WAC) foi conhecido como um movimento de reforma do ensino superior estadunidense que teve como objetivo inserir componentes curriculares de escrita nos semestres iniciais dos cursos de graduação, em resposta à entrada de alunos anteriormente excluídos para o ensino superior A primeira iniciativa foi a oferta de programas de escrita nas disciplinas para graduandos das engenharias e sua área de atuação, tendo em vista atender às queixas dos empregadores desses profissionais (SILVA; REINALDO, 2016, p.143). 
principal de aprimorar a escrita dos alunos. Contudo, o WAC também se propõe a melhorar o aprendizado dos conteúdos específicos de cada curso. A metodologia tem base na teoria Vygotskyana, pois defende que, ao escrever ensaios, diários, artigos, o estudante constrói o seu conhecimento e se torna agente de sua aprendizagem (SILVA; REINALDO, 2016, p. 143). O método - adaptado para as aulas de FEMA consiste em uma leitura prévia sobre o assunto antes da aula expositiva. Logo depois da aula, o aluno produz seu texto, com a liberdade de consultar o material e trocar ideias com os colegas, sintetizando o assunto da aula. 0 texto, então, recebe correções de língua portuguesa (gramática e estilo) e do conteúdo estudado e o aluno tem a devolutiva, ou seja, o feedeback avaliativo do professor sobre os problemas a serem superados. As produções dos discentes, realizadas na metodologia WAC, puderam ser utilizadas como material de apoio nas avaliações individuais e trabalhos em grupos.

O método PBL - Problem Based Learning (Aprendizagem Baseada em Problemas) é um método no qual os alunos aprendem através da solução de problemas reais e se tornam responsáveis pelo seu próprio aprendizado, devendo refletir sobre suas experiências (HMELO-SILVER, 2004). O PBL, aplicado na disciplina FEMA, buscou ajudar os alunos a desenvolver estratégias para solucionar problemas e a construir conhecimento a partir das soluções desenvolvidas para que, em outras circunstâncias, tal conhecimento possa ser aplicado e replicado. Essa metodologia foi aplicada em propostas avaliativas que verificaram competências procedimentais na resolução de situações-problema e produção de trabalhos práticos.

O PBL e o WAC foram aplicados em algumas aulas de FEMA, complementando o trabalho realizado, principalmente com a metodologia Peer instruction, com a finalidade de contribuir com a avaliação formativa e aprendizagem discente, foco da discussão deste artigo.

\section{MÉTODOS E TÉCNICAS}

A pesquisa-ação corresponde à estratégia metodológica utilizada na formação científico-pedagógica dos professores da Universidade Tuiuti do Paraná e envolveu a participação desses profissionais em grupos de estudo e sua integração no processo de indagação e diálogo sobre as práticas pedagógicas. Essa concepção de pesquisa contribui para o desenvolvimento profissional, pois, ao envolver o coletivo de professores da universidade no processo de problematização e desenvolvimento da pesquisa, eleva-se o nível de consciência coletiva para a compreensão e solução dos problemas da prática pedagógica e os educacionais mais amplos (FONTANA; MENDES; PRESTES, 2018, p.34).

A pesquisa-ação, conforme Thiollent (1998, p. 15), tem como compromisso a ação sobre uma problemática que merece investigação para ser elaborada e conduzida. A problemática definida no grupo de pesquisa refere-se às repercussões do uso das metodologias ativas na aprendizagem discente na educação superior. A partir desse questionamento inicial, os professores realizaram investigações em suas salas de aula sobre sua prática pedagógica e buscam referências bibliográficas para compor o marco teórico de suas ações práticas. No caso da presente investigação, definiu-se para análise o campo de observação, a amostragem e representatividade quanti-qualitativa, registro dos dados em diário de campo e formulários (planilha de acompanhamento diagnóstico das avaliações).

A pesquisa foi realizada durante o primeiro semestre letivo do ano de 2019, na disciplina de Fundamentos da Engenharia Mecânica Automobilística (FEMA), de 80h, com 34 alunos matriculados. 0 problema que demandou a pesquisa na disciplina foi a dificuldade de alguns alunos em relação ao conteúdo ministrado e a falta de estudo e leitura da maioria dos alunos sobre o conteúdo programado para as aulas, dificultando a aprendizagem e o trabalho com metodologias ativas que requerem a leitura prévia. 
O desenvolvimento das aulas contou com a utilização de diferentes metodologias ativas, conforme explicitado anteriormente, para promover maior interação entre os alunos e o professor, motivá-los para presença em sala de aula e melhorar a aprendizagem geral da turma. 0 uso da metodologia Peer Instruction foi intensificado na disciplina com o objetivo de realizar as atividades avaliativas na perspectiva diagnóstica e contínua, pois permite aos alunos interagirem e compartilharem conhecimentos, e o feedback do professor para revisão do conteúdo ministrado. Essa metodologia, como ressalta Mazur (2015), foge do conceito tradicional de ministrar uma aula detalhada, mas consiste em apresentar de forma curta os pontos principais do conteúdo, seguido de pequenas questões conceituais para promover a interação entre os estudantes e focar a atenção deles nos conceitos fundamentais da disciplina. Entende-se que a maior potencialidade do método é obtida, quando as questões conceituais apresentadas pelo professor apresentam uma distribuição de frequências das respostas dos alunos entre $35 \%$ e $70 \%$ de acertos. Nesse caso, a turma é dividida em pequenos grupos, em média três alunos, para discutirem a questão conceitual. É nesse momento que acreditamos ocorrer a valiosa aprendizagem entre pares.

A partir de estudos fundamentados em Luckesi (2018, p.28), compreende-se que avaliar implica coleta, análise e síntese dos dados sobre o objeto da avaliação, acrescido de uma atribuição de valor ou qualidade. 0 valor ou qualidade atribuído ao objeto conduz a uma tomada de posição que orienta o professor para uma decisão nova em favor de melhorar a aprendizagem sobre o objeto da avaliação.

Para organizar o processo avaliativo na perspectiva formativa, diagnóstica e contínua na disciplina de FEMA, foram realizados os seguintes procedimentos na prática pedagógica:

a) Publicação dos conteúdos e material de apoio no ambiente virtual de aprendizagem (AVA) da Universidade.

b) Apresentação do tema com definição do sistema automotivo a ser estudado, discussão em sala de aula em função de situações particulares de aplicações e existência de casos inovadores ou pouco utilizados.

c) Descrição dos componentes que integram o sistema automotivo.

d) Interação dos sistemas automotivos entre si.

e) Aplicação prática dos conceitos estudados em sala de aula pela resolução de problemas, ou seja, pela construção de sistemas prototipados.

f) Investigação teórica com aplicação prática feita como tarefa extraclasse (Estudo Dirigido).

g) Realização de atividade em grupos, com até cinco estudantes, para a resolução de situação-problema, utilizando-se a adaptação da metodologia Peer Instruction combinada com a metodologia Problem Based Learning.

h) Elaboração de textos, em alguns encontros, pelos alunos, para sistematização do conteúdo da aula, baseados na metodologia Writing Across the Curriculum.

Em síntese, nas aulas de FEMA, os conteúdos foram publicados previamente, segundo o planejamento, para possibilitar ao aluno a oportunidade de ler e pesquisar sobre os temas de estudos. A combinação e adaptação das metodologias ativas Peer instruction, Problem Based Learning e Writing Across the Curriculum, utilizadas nas propostas de trabalhos em grupos, promoveu a interação entre pares para a pesquisa teórica, a execução prática por meio da resolução da situação-problema e a escrita, contem- 
plando a sistematização dos conteúdos e formatação própria para apresentação oral. No desenvolvimento das tarefas em grupos, os discentes aprenderam a importância de apoiar quem está com dificuldade no entendimento dos temas e descobriram quais são suas habilidades e facilidades para realizar tarefas dentro do grupo de trabalho. Essas aprendizagens foram relevantes para o desenvolvimento de atitudes de cooperação e de compartilhamento de conhecimentos.

Os registros das atividades avaliadas foram organizados em uma planilha de acompanhamento diagnóstico da turma e possibilitou observar importantes dados para o trabalho avaliativo do professor, tais como, as dificuldades de aprendizagem, faltas na disciplina, o possível abandono de curso e, principalmente, a identificação dos discentes com dificuldade de aprendizagem nos conteúdos trabalhados.

\section{RESULTADOS E DISCUSSÃO}

Os resultados observados na pesquisa-ação desenvolvida na disciplina de FEMA e que contribuíram para o uso diagnóstico dos resultados da avaliação, para tomada de decisão em favor da elevação da aprendizagem de todos os discentes, no processo de construção e apropriação de conhecimentos foram organizados para sua apresentação em relação: 1. Metodologia desenvolvida na prática pedagógica da disciplina de FEMA; 2. Análise da planilha de acompanhamento diagnóstico para a avaliação formativa, aprendizagem e organização da prática pedagógica; 3. Revisão e aperfeiçoamento do processo de pesquisa-ação.

\subsection{Metodologia desenvolvida na prática pedagógica da disciplina de FEMA}

A metodologia empregada propiciou um sistema de avaliação aula a aula, sendo explicitado, para o acompanhamento dos alunos, a construção das suas aprendizagens por meio do registro de notas em uma planilha das avaliações. As avaliações foram previamente planejadas, tendo como referência o desenvolvimento de competências e habilidades dos discentes previstas no plano de aula da disciplina. Foram realizadas vinte e duas (22) avaliações de questionários de múltipla escolha com cinco (5) questões cada uma e gabarito de respostas com cinco (5) combinações de respostas para cada questão. 0 total das avaliações aplicadas à turma foi de 110 questões com 440 distratores e 550 combinações de respostas, em questionários avaliativos tradicionais.

Além de questões objetivas e reflexivas, a disciplina também trabalhou seis (6) tarefas desenvolvidas extraclasse, que foram avaliadas, corrigidas e registradas em forma de pesquisas teóricas, exercícios e construção de protótipos.

O uso da planilha de acompanhamento diagnóstico ${ }^{7}$, conforme mostra a figura 1, teve a finalidade de 0 professor analisar os resultados dos processos de aprendizagem de seus discentes para (re)planejar o ensino.

\footnotetext{
7 A figura mostra as avaliações realizadas, no início do semestre, sendo elas: individuais de múltipla escolha (branco), avaliações em grupo (cinza), avaliações resultantes do PI (rosa), avaliação prática (verde). As indicações em amarelo mostram os alunos ausentes no dia da avaliação, o que comprova a boa assiduidade dos alunos.
} 
Figura 1 - Exemplo de planilha de acompanhamento diagnóstico da avaliação da aprendizagem

Data da avaliação

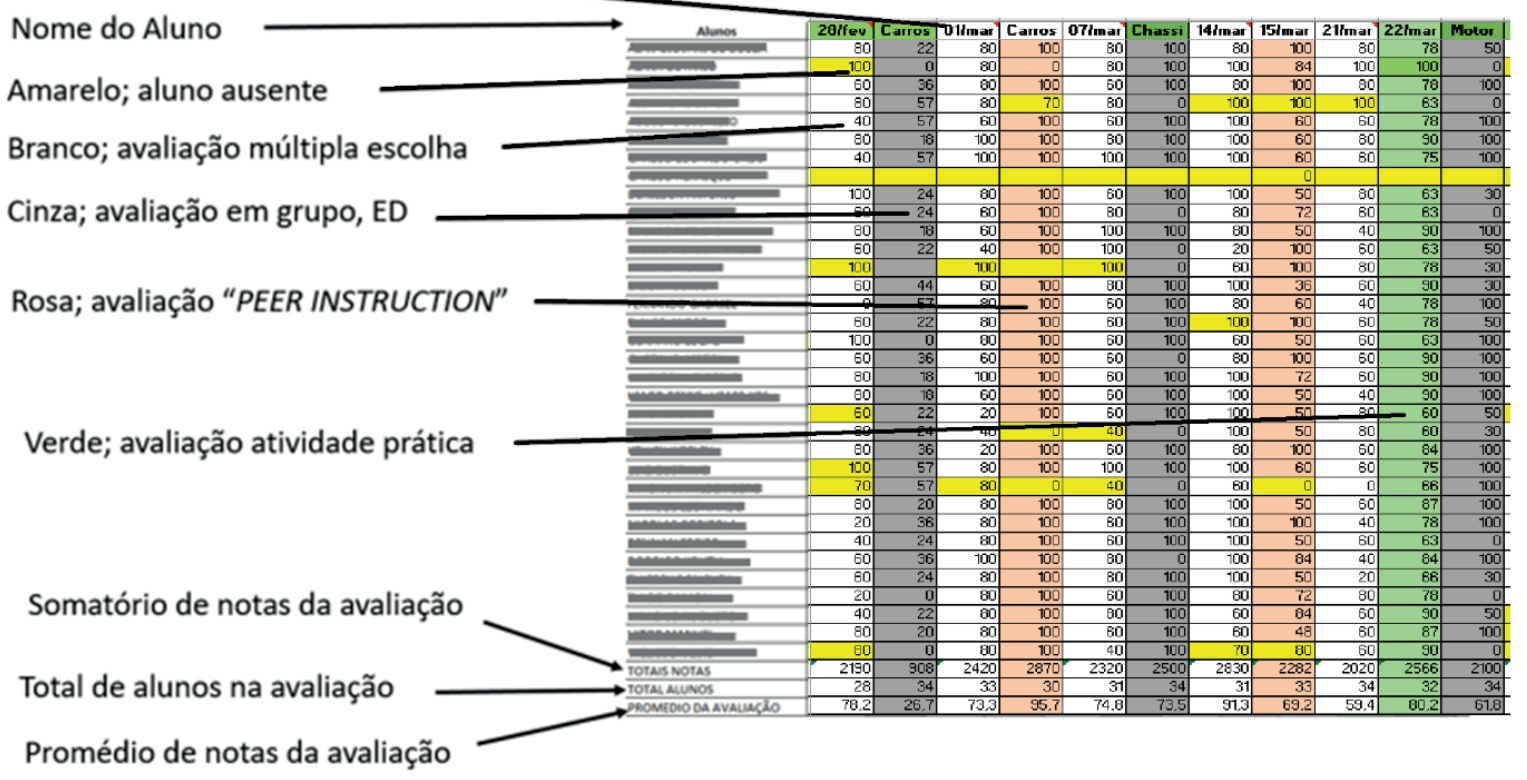

Fonte: Autoria própria (2019)

A planilha para o acompanhamento diagnóstico do professor reuniu os dados das avaliações das aprendizagens individuais em relação aos conteúdos trabalhados. Em cada aula, os discentes receberam a devolutiva de seu aprendizado e, quando necessário, foi realizada a revisão dos conteúdos e conceitos não aprendidos. Nessas situações, os conteúdos foram expostos e analisados entre todos, como uma grande mesa redonda de análise e discussão. Essa proposta de feedback contínuo ajudou o discente no automonitoramento, isto é, refletir sobre o desempenho de sua aprendizagem e o professor a revisar o ensino e as metodologias adotadas.

A planilha de acompanhamento diagnóstico também contribuiu para a visualização da atitude do aluno em relação à presença e participação em sala de aula, ao comprometimento com as datas de entrega de trabalhos e em relação à qualidade dos trabalhos e das avaliações realizadas. Possibilitou ao discente e ao docente a visualização das aulas e dos conteúdos ministrados, registrados nas colunas das avaliações. Por exemplo: cada módulo teve 4 (quatro) avaliações de múltipla escolha individuais, uma avaliação Peer Intruction e uma avaliação de Estudo Dirigido (tarefa extraclasse). Portanto, foi um instrumento que contribuiu para a organização e para a análise da qualidade dos dados, subsidiando as decisões do professor para a intervenção pedagógica.

\subsection{Análise da planilha de acompanhamento diagnóstico para a avaliação formativa, aprendizagem e organização da prática pedagógica}

As avaliações realizadas continuamente, durante todo o semestre, foram acompanhadas por meio da planilha de acompanhamento diagnóstico do desempenho discente, que possibilitou o feedback sobre o processo de ensino-aprendizagem e as intervenções para os problemas detectados. 0 resultado da avaliação geral da turma foi a média de 72 , alcançando uma aprovação direta de 25 alunos, o que correspondeu a $73 \%$ dos alunos.

Constatou-se a assiduidade de $91 \%$ dos discentes ao longo do semestre, com frequência assídua de 31 alunos, considerando o total de 34 matriculados. Infere-se que o uso das metodologias ativas con- 
tribuiu para a presença participativa e colaborativa entre colegas em sala de aula e, por consequência, a motivação e melhora da aprendizagem geral da turma. Observou-se, durante o acompanhamento diagnóstico, até o final do $1^{\circ}$ semestre de 2019, que a utilização da metodologia PI associada ao PBL e WAC contribuiu para elevar a média da maioria dos discentes em avaliações do mesmo conteúdo, ou seja, as notas obtidas nas avaliações realizadas em pares e na resolução de problemas práticos, para a maioria dos alunos, revelaram uma boa aprendizagem dos conteúdos.

É importante ressaltar que, para algumas avaliações individuais de múltipla escolha, o material de consulta foi o WAC do aluno - o texto produzido ao final da aula que contém a síntese dos tópicos estudados. Essa estratégia metodológica contribuiu para o discente organizar suas anotações e desenvolver habilidades de escrita e interpretação de textos.

\subsection{Revisão e aperfeiçoamento do processo de pesquisa-ação}

A condução da disciplina FEMA, utilizando a combinação de várias metodologias, possibilitou verificar a dificuldade da aplicação dos conteúdos de cálculo diferencial e integral, para a resolução de problemas práticos relativos aos fundamentos da engenharia mecânica. Para sanar esse problema, foi planejada uma sequência de aulas conjuntas com a professora que ministra a disciplina de Cálculo Diferencial e Integral e que também participa do grupo de pesquisa-ação. Nessa proposta, dois aspectos foram observados: a reação positiva da turma, passando a participar ativamente das aulas de cálculo e o entendimento da necessidade da interdisciplinaridade entre disciplinas do curso. Contextualizar e integrar os conteúdos se mostrou, para os docentes e discentes, ser uma metodologia de suma importância, pois uma teoria sem contextualização gera uma lacuna, quando se faz necessária a aplicação do conhecimento em situações práticas da profissão, como foi enfatizado na proposta metodológica da disciplina de FEMA.

\section{CONSIDERAÇÕES FINAIS}

A avaliação é uma prática complexa, principalmente quando tem o objetivo de subsidiar o docente a tomar decisões ajustadas para garantir que todos aprendam. Após o exposto sobre a pesquisa-ação na disciplina FEMA, pode-se concluir que não é suficiente uma única metodologia para promover a aprendizagem geral de uma turma, pois os discentes não são iguais e não aprendem da mesma maneira. Dessa forma, pode-se afirmar que a combinação de metodologias Peer Instruction, Writing Across the Curriculum e Problem Based Learning com o foco na avaliação formativa da aprendizagem possibilitou vários e diferentes caminhos para o aluno aprender e desenvolver suas competências e habilidades sobre conceitos da disciplina, resolução de problemas práticos e nas atitudes de cooperação e compartilhamento de conhecimentos. Conforme o exemplo da planilha de notas, verificam-se boas médias nas avaliações realizadas em pares, quando utilizado o $\mathrm{Pl}$ e o $P B L$ para a resolução de problemas práticos, assim como nas provas individuais em que foi utilizado o WAC. Portanto, comprova-se a produtividade pelo resultado da avaliação geral da turma, cuja média foi 72 , com a aprovação direta de $73 \%$ dos alunos, situação diferente de outros semestres, em que era utilizada a metodologia tradicional. Entende-se que as metodologias ativas favoreceram, pelas suas características, o feedback necessário para a efetivação da avaliação formativa e a melhoria da aprendizagem dos discentes.

As práticas avaliativas contribuíram para o discente monitorar-se e elevar sua aprendizagem e subsidiaram o professor para planejar as aulas com diferentes metodologias que articularam o conhecimento teórico-prático dos conteúdos da disciplina. Pretende-se dar continuidade à pesquisa-ação e, principalmente, realizar a combinação com outros componentes curriculares, a fim de se intensificar a interdisciplinaridade e a contextualização dos conteúdos, para efetivar a aprendizagem discente, no curso de Engenharia Mecânica. 


\section{REFERÊNCIAS}

BOLDARINE, Rosaria De Fátima; BARBOSA, Raquel Lazzari Leite. ANNIBAL, Sérgio Fabiano. Tendências da produção de conhecimento em avaliação das aprendizagens no Brasil (2010-2014). Estudos de Avaliação Educacional, São Paulo, v. 28, n. 67, p. 160-189, jan./abr. 2017.

FONTANA, Maria Iolanda; MENDES, João Roberto; PRESTES, Irene Carmen Picone. Formação continuada dos professores da universidade: contribuições da pesquisa-ação para a inovação pedagógica. Revista Tuiuti: Ciência e Cultura, Curitiba, v. 56, n. 5, p.27-47, 2018.

GARCIA, J. Avaliação e aprendizagem na educação superior. Estudos de Avaliação Educacional, São Paulo, v. 20, n. 43, p. 201-213, maio/ago. 2009.

HMELO-SILVER, Cindy E. Problem-Based Learning: What and How Do Students Learn? Educational Psychology Review, V. 16, n. 3, p. 235-266, September, 2004.

HOFFMANN, Jussara. Avaliação mediadora: uma prática em construção da pré-escola à universidade. Porto Alegre; Editora Mediação, 2009.

LUCKESI, Cipriano Carlos. Avaliação das aprendizagens escolar. 19. ed. São Paulo: Cortez, 2008.

LUCKESI, Cipriano Carlos. Avaliação em educação: questões epistemológicas e práticas. São Paulo: Cortez, 2018.

MAZUR, E. Peer Instruction: a revolução da aprendizagem ativa. Porto Alegre: Penso, 2015.

MÜLLER, Maykon Gonçalves; ARAUJO, Ives Solano; VEIT, Eliane Angela; SCHELL, Julie. Uma revisão da literatura acerca da implementação da metodologia interativa de ensino Peer Instruction (1991 a 2015). Revista Brasileira de Ensino de Física, v. 39, n. 3, p. e3403, 2017.

SILVA, Elizabeth Maria da; REINALDO, Maria Augusta Gonçalves de Macedo. Escrita disciplinar: contribuições para o ensino de Língua Portuguesa na graduação. Ilha do Desterro v. 69, n. 3, p. 141-155, Florianópolis, set/dez 2016.

SILVA, Natália Luiza; MENDES, Olenir Maria. Avaliação formativa no ensino superior: avanços e contradições. Avaliação, Campinas; Sorocaba, SP , v. 22, n. 1, p. 271-297, mar. 2017.

THIOLLENT, Michel. 1998. Metodologia da pesquisa-ação. 8.ed. São Paulo: Cortez, 1998.

VASCONCELLOS, Celso dos Santos. Avaliação da aprendizagem: práticas de mudança por uma práxis transformadora. 11. ed. São Paulo: Libertad, 2010.

VILLAS BOAS, Benigna Maria de Freitas. Virando a escola do avesso por meio da avaliação. 2. ed. Campinas: Papirus, 2009. 